\begin{tabular}{|c|c|}
\hline Title & $\begin{array}{l}\text { Characteristics of Symptomatic Intracerebral Hemorrhage in Patient Receiving Direct Oral A nticoagulants: } \\
\text { Comparison with W arfarin }\end{array}$ \\
\hline Author(s) & $\begin{array}{l}\text { Kawabori, Masahito; Niiya, Y oshimasa; Iwasaki, Motoyuki; Mabuchi, Shoji; Ozaki, Hiroyuki; Matsubara, Koji; } \\
\text { Houkin, Kiyohiro }\end{array}$ \\
\hline Citation & $\begin{array}{l}\text { Journal of stroke \& cerebrovascular diseases, } 27(5), 1338.1342 \\
\text { https://doi.org/10.1016/.jstrokecerebrovasdis.2017.12.020 }\end{array}$ \\
\hline Issue Date & 201805 \\
\hline Doc URL & http:/hdl.handle.net/2115/73805 \\
\hline Rights & $\begin{array}{l}\text { () 2018. This manuscript version is made available under the CC-BY-NC-ND } 4.0 \text { license } \\
\text { http://creativecommons.org/icenses/by-nc-nd/4.0/ }\end{array}$ \\
\hline Rights(URL) & http://creativecommons.org/icenses/by-nc-nd/4.0/ \\
\hline Type & article (author version) \\
\hline File Information & JStrokeCerebrovascDis27_1338.pdf \\
\hline
\end{tabular}

Instructions for use 


\section{Characteristics of Symptomatic Intracerebral Hemorrhage in Patient Receiving Direct Oral Anticoagulants: Comparison with Warfarin}

Masahito Kawabori, $\mathrm{MD}, \mathrm{PhD}^{1,3)}$, Yoshimasa Niiya $\mathrm{MD}, \mathrm{PhD}^{1)}$, Motoyuki Iwasaki MD,

$\mathrm{PhD}^{1)}$, Shoji Mabuchi MD, $\mathrm{PhD}^{1)}$, Hiroyuki Ozaki, $\mathrm{BS}^{2)}$, Koji Matsubara $\mathrm{BS}^{2)}$, Kiyohiro Houkin $\mathrm{MD}, \mathrm{PhD}^{3)}$

1) Department of Neurosurgery, Otaru General Hospital

2) Department of Pharmacology, Otaru General Hospital

3) Department of Neurosurgery, Hokkaido University Graduate School of Medicine

Key words : DOAC, intracerebral hemorrhage, warfarin, Stroke

Grant support: No financial support to disclose

Shortened title: Characteristics of ICH with Warfarin and DOAC

\section{Corresponding Author:}

Masahito Kawabori

Email: masahitokawabori@yahoo.co.jp

Tel: 011-706-5987

Fax: 011-708-7737

Department of Neurosurgery, Hokkaido University Graduate School of Medicine

Kita 15, Nishi 7, Kita-ku, Sapporo, Hokkaido, 060-8638, Japan 
Background: Direct oral coagulants (DOAC) have been shown to decrease the frequency of intracerebral hemorrhage (ICH) compare to warfarin. However, the precise characteristics, such as the size and locations of the hemorrhage, and outcome and onset time of ICH in patient taking DOAC are not fully elucidated.

Methods: We retrospectively analyzed the characteristics of symptomatic ICH patients taking either DOAC or warfarin between January 2012 and December 2015.

Results: Out of consecutive 400 ICH patients, 15 patients were DOAC-ICH and 24 patients were warfarin-ICH. DOAC-ICH was seen in 6 patients with $10 \mathrm{mg}$ rivaroxaban, 5 patients with $15 \mathrm{mg}$ rivaroxaban, one patient with $10 \mathrm{mg}$ apixaban, $5 \mathrm{mg}$ apixaban, 30mg edoxaban, and $60 \mathrm{mg}$ edoxaban. Prothrombin time were well controlled in most of the warfarin-ICH patients (83.3\%). The locations of ICH were similar in the both groups, however median ICH volume was significantly smaller in DOAC-ICH than warfarin-ICH $(\mathrm{p}<0.01)$ and ICH around basal ganglia seemed to show great difference between the groups. DOAC-ICH patients showed better neurological outcome at the time of discharge compared to warfarin patients $(\mathrm{p}<0.01)$ and the ratio of good prognosis was significantly higher in the DOAC-ICH patients compare with warfarin-ICH patients $(\mathrm{p}<0.01$ ). The onset of warfarin-ICH were frequently seen in the morning and evening, while DOAC-ICH did not show any specific onset time.

Conclusion: Patients with DOAC-ICH showed smaller ICH volume and better clinical outcomes compared with warfarin-ICH, and DOAC-ICH did not show any specific onset peak. 
Intracerebral hemorrhage (ICH) is one of the most serious complications of oral anticoagulation, with high in-hospital mortality. ${ }^{1} \quad$ In large phase 3 trials, patients taking Direct oral antagonist coagulants (DOAC) showed almost a half frequency of ICH compared to warfarin, despite the similar efficiency. ${ }^{2}$ However, beside from the data of hemorrhagic frequency, few data are available on the precise clinical and neurological characteristics of DOAC-ICH, such as severity of the hemorrhage and neurological outcome. There is widespread concern that, without any currently available specific antidotes for most of the DOAC, DOAC-ICH might have larger ICH volumes and worse clinical outcomes than warfarin-ICH., ${ }^{3,4}$ Furthermore, DOAC are shown to have shorter plasma half-life compare to warfarin and its magnitude of the serological change are concerned to be the cause of hemorrhage, but the onset timing of hemorrhage is also unknown. In this study, we aim to elucidate the characteristics of DOAC-ICH in comparison with warfarin-ICH patients who were admitted to our single local comprehensive stroke center.

Methods

Ethical statement

This study was conducted in accordance with the guideline principles of the Declaration of Helsinki and was approved by the local ethics committee of Otaru General Hospital. All patients or their families gave either oral or written informed consent to participate in this study.

Patients

We retrospectively analyzed symptomatic ICH patient who were taking either warfarin or DOAC at the time of admission between January 2012 and December 2015. The following clinical factors and outcome at the time of hospital discharge were examined 
retrospectively; age; sex; previous history of ischemic stroke/ICH, underlying illness; type of NOAC; dose of NOAC/warfarin; concurrent anticoagulation; blood test results; predictive scores for the risk of bleeding (HAS-BLED, ATRIA, ORBIT); number of positive T2* on magnetic resonance (MR) imaging; ICH-related information (location, hematoma volume, surgical treatment), and clinical prognosis at the time of discharge (modified Rankin Scale; mRS). Hematoma volume was calculated with the following formula using measurements of a plain computed imaging (CT) image of the brain: maximum length of an ellipsoid representing intracerebral hemorrhage $\mathrm{x}$ width perpendicular to maximum length $\mathrm{x}$ thickness of hematoma $\mathrm{x} 0.5$.

Statistical analysis

All data were expressed either as median (interquartile range [IQR]) or average \pm standard deviation (SD). Continuous data were compared by unpaired t-test or $X^{2}$ test between two groups and cluster analysis were done by Kolmogorov Smirnov test. Values of $\mathrm{P}<0.05$ were considered statistically significant.

Results

Out of 400 consecutive ICH patients, we have encountered 15 patients (3.8\%) with NOAC-ICH and 24 patients (6.0\%) with warfarin-ICH. The median age of the patients was slightly higher in the warfarin-ICH group (81 years old) compare to DOAC-ICH group (74 years old) $(\mathrm{p}=0.05)$, but there was no sex difference. The patient with warfarin-ICH had taken $2.9 \pm 1.0 \mathrm{mg}$ of warfarin at the time of admission and DOAC-ICH was seen in 6 patients with $10 \mathrm{mg}$ rivaroxaban, 5 patients with $15 \mathrm{mg}$ rivaroxaban, one patient with $10 \mathrm{mg}$ apixaban, one patient with $5 \mathrm{mg}$ apixaban, one patient with 30mg edoxaban, and one patient with $60 \mathrm{mg}$ edoxaban. Prothrombin time international normalized ratio (PT-INR) of the warfarin-ICH was 2.6 (2.1-2.9) and was 
well controlled that in 20 out of 24 warfarin-ICH patients (83.3\%) were under 3.0, while PT-INR in the DOAC-ICH were as low as $1.2(1.1-1.8)$ and was significantly lower than warfarin-ICH $(\mathrm{P}<0.01)$. Coexisting illness were examined between the patients, and the rate of anemia (56.5\% vs $13.3 \%, \mathrm{P}<0.01)$ and chronic renal failure $(17.4 \%$ vs $0.0 \%$, $\mathrm{P}<0.04)$ were higher in the warfarin-ICH group, however, other coexisting illness such as congestive heart failure (warfarin vs DOAC; $29.2 \%$ vs 26.6 ), diabetes (29.2\% vs 20.0\%), hypertension (87.5\% vs $90.0 \%$ ), low total cholesterol (15.4\% vs $26.3 \%)$, previous history of ischemic stroke (25.0\% vs $26.7 \%)$, previous history of ICH (8.3\% vs 6.7\%), and number of positive T2* on MR imaging (1, [IQR 1-7] vs 2, [IQR 2-11]) did not show statistical difference between the groups (Table 1). Concomitant use of antiplatelet drugs were also similar between the groups $(\mathrm{p}=0.15)$, which 9 patients were seen in the warfarin-ICH (7 patients with $100 \mathrm{mg}$ of aspirin, 1 patient with $100 \mathrm{mg}$ of ticlopidine, and 1 patient with $100 \mathrm{mg}$ of aspirin and $200 \mathrm{mg}$ of cilostazol), and two patient were seen in the DOAC-ICH group (1 patient with $100 \mathrm{mg}$ of aspirin, and 1 patient with $75 \mathrm{mg}$ of clopidogrel). Because the warfarin-ICH patients were older, and had higher rate of anemia and renal failure, all the predictive scores for the risk of bleeding, such as HAS-BLED, ATRIA, ORBIT were shown to be statistically higher in the warfarin-ICH group.

The locations of ICH were similar in the both groups, such as cerebral cortex, basal ganglia, and cerebellar nucleus, which resemble hypertensive cerebral hemorrhage. (figure 1) However the median ICH volume was significantly smaller in DOAC-ICH (5.9 mL, IQR 3.5 - $12.3 \mathrm{~mL})$ compared with warfarin-ICH (27.0 mL, IQR7.3 - 39.3 $\mathrm{mL})(\mathrm{p}<0.01)$. It seemed that the size of ICH around basal ganglia seemed to be much smaller in the NOAC-ICH compare to the Warfarin-ICH. Warfarin-ICH showed 
higher tendency of emergency surgery for evacuation of ICH (50\% vs $26 \%$; $p=0.15$ ) and hospital administration days (31 days vs 20 days; $\mathrm{p}=0.20$ )(Table 2). DOAC-ICH patients showed better neurological outcome at the time of discharge (mRS 2, IQR 1-4) compared to warfarin-ICH patients (5, IQR 4-5) $(\mathrm{p}<0.01)$, and the ratio of good prognosis (mRS 0-2 at discharge) was also significantly higher in the DOAC-ICH patients (53\%) compare to warfarin-ICH patients $(12.5 \%)(\mathrm{p}<0.01)$. When comparing the onset of ICH between warfarin and DOAC groups, there were several patients whose onset time were unclear because the patient may live by him/herself, but according to the data available, warfarin-ICH showed bimodal onset peak in the morning and evening, while DOAC-ICH did not show any specific onset time, however cluster analysis using Kolmogorov Smirnov test did not show any difference $(\mathrm{P}=0.38)$ (figure 2).

\section{Discussion}

Our results from this single comprehensive stroke center show that DOAC-ICH is associated with smaller ICH volume and better clinical outcome than warfarin-ICH. There were no differences at the site of the hemorrhage between the groups, but hemorrhage around basal ganglia seemed to be smaller in the DOAC-ICH. Warfarin-ICH showed bimodal onset peak while NOAC-ICH were seen throughout the day and did not show any peak onset timing.

Beside from the large amount of evidence regarding the frequency of the $\mathrm{ICH}$ in patient taking DOAC and warfarin, we have found only 2 previous reports on the clinical and radiologic characteristic of DOAC-ICH compared with warfarin-ICH. ${ }^{5,6}$ Hagii et al. have included 5 patients with rivaroxaban-associated ICH which was shown to be 
smaller than warfarin-ICH, ${ }^{5}$ and Wilson et al. compared 11 patients with DOAC-ICH and 52 patients with warfarin-ICH, and warfarin use was found to be independently associated with larger ICH volume. ${ }^{6}$ Our data will further confirm the fact that DOAC-ICH were smaller and clinically better compare to the warfarin-ICH. One of the interesting point of our data is that most of the warfarin-ICH patients in this trial met standard PT-INR range which $83.3 \%$ of the patients showed PT-INR under 3.0, and this clearly denotes that usage of warfarin under appropriate range may not protect patient from ICH in patients with warfarin, and DOAC may also be superior in this point. However, the mechanism leading to smaller hemorrhage volume in DOAC-ICH compared with warfarin-ICH needs further clarification. One of the explanations is the different functional mechanism between the warfarin and DOAC regarding the high level of tissue factor (TF) around blood vessels in the brain affecting extrinsic and/or TF-dependent coagulation pathway. ${ }^{7}$ When the vessels are damaged, cells presenting TF around the vessel come into contact with factor VII (FVII) and active factor VII (FVIIa) in the blood soon after the vascular injury, and TF binds both FVII and FVIIa with high affinity. TF-FVIIa activates factor X (FX) both directly (extrinsic pathway) and indirectly via activation of factor IX, leading to fibrin formation. ${ }^{8}$ Warfarin which is the vitamin $\mathrm{K}$ antagonist inhibit the vitamin $\mathrm{K}$ conversion cycle, resulting in reducing hepatic production of partially decarboxylated vitamin K-dependent coagulation proteins II, VII, IX, and X, concomitant with reduced coagulant activity. ${ }^{9}$ Thus, warfarin reduces the extrinsic coagulation pathway and subsequent fibrin formation by reducing the amount of factor VII interacts with TF. On the other hand, DOAC specifically and competitively inhibit FXa (rivaroxaban, apixaban, and edoxaban) or thrombin (dabigatran), and reduce the thrombin cascade during the downstream 
propagation phase of coagulation or thrombin activity compare to warfarin. $^{10,} 11$ DOAC is considered not to affect the initial factor VII and VIIa interaction with TF, and may provide an explanation for the smaller hemorrhage volume for DOAC-ICH. Fleck et al. have reported that the gray matter showed higher amount of TF staining than white matter and this high amount of TF existence may contribute smaller hemorrhage volume. ${ }^{9} \quad$ This finding is concomitant with our result which volume of hemorrhage around basal ganglia is especially reduced in DOAC-ICH compare to warfarin-ICH. In this report, we did not find difference in location of the hemorrhage. This result indicates that there is no DOAC interaction with the specific brain vessel to cause vessel injury. Because of the hemorrhage size, it is quite reasonable to explain that smaller $\mathrm{ICH}$ is associated with better clinical outcome. The difference was quite large that median mRS at the time of discharge were 2 in the DOAC-ICH compare to 5 in the warfarin-ICH. This result is compatible with the past literature reported DOAC-ICH group showed lesser ICH volume and better clinical outcome at the time of discharge compared to warfarin-ICH. ${ }^{5,6}$

In this report, we have shown that DOAC-ICH did not show any specific timing of onset, while warfarin-ICH seems to have bimodal peak in the morning and evening as previously reported ${ }^{12}$. This was different from our previous prediction that DOAC-ICH have shorter plasma half-lives which may cause abrupt elevation of the plasma concentration and resulting ICH presumably in the morning or the evening when the DOAC are being taken.

We understand that there are not a few limitations seen in this study. First, we could not find the whole population of the patient taking warfarin and DOAC, since most of the patients receive drugs from the local clinics. This will make it impossible to find 
the accurate ICH ratio of the patient who are taking these drugs. However, several larger cohorts and randomized trials have shown the frequency of ICH and we can probably estimate that ICH ratio should be lower in DOAC-ICH. Second, our data showed that warfarin-ICH patients showed slightly higher age and worse renal functions which may affect the size of the hemorrhage and clinical outcome. We may need to adjust the patient background using such as cross-sectional observational study, however it was quite difficult to do this in our small number of patient, and this will need further larger cohort. Third, the data of the onset timing can only be acquired from the patient or family and the data may not be accurate that hemorrhage may be occurred before or after the patient felt illness, since smaller hemorrhage may take time to show clinical symptom, or other illness may lead to the hemorrhage by elevating blood pressure. Fourth, because of the lack of analysis regarding cost effectiveness and others analysis such as QALY, the data does not provide the evidence that the DOAC usage were superior to that of warfarin in all of the patients who requires anti-coagulant therapy. Cerebral hemorrhage patient needs extensive social support which require high cost, but on the other hand, DOAC itself is much expensive than warfarin. Further evaluation using meta-data regarding the cost reduction rate of may be necessary to draw conclusion in the future.

DOAC are associated with reduced ICH volume and better clinical outcome compare to the warfarin, and DOAC did not seem to associate with specific onset timing. 
1. Dowlatshahi D, Butcher KS, Asdaghi N, Nahirniak S, Bernbaum ML, Giulivi A, et al. Poor prognosis in warfarin-associated intracranial hemorrhage despite anticoagulation reversal. Stroke. 2012;43:1812-7.

2. Ruff CT, Giugliano RP, Braunwald E, Hoffman EB, Deenadayalu N, Ezekowitz MD, et al. Comparison of the efficacy and safety of new oral anticoagulants with warfarin in patients with atrial fibrillation: a meta-analysis of randomised trials. Lancet. 2014;383:955-62.

3. Schulman S, Majeed A. The oral thrombin inhibitor dabigatran: strengths and weaknesses. Semin Thromb Hemost. 2012;38:7-15.

4. Ansell J. New oral anticoagulants should not be used as first-line agents to prevent thromboembolism in patients with atrial fibrillation. Circulation. 2012;125:165-70; discussion 70 .

5. Hagii J, Tomita H, Metoki N, Saito S, Shiroto H, Hitomi H, et al. Characteristics of intracerebral hemorrhage during rivaroxaban treatment: comparison with those during warfarin. Stroke. 2014;45:2805-7.

6. Wilson D, Charidimou A, Shakeshaft C, Ambler G, White M, Cohen H, et al. Volume and functional outcome of intracerebral hemorrhage according to oral anticoagulant type. Neurology. 2016;86:360-6. 
7. Fleck RA, Rao LV, Rapaport SI, Varki N. Localization of human tissue factor antigen by immunostaining with monospecific, polyclonal anti-human tissue factor antibody. Thromb Res. 1990;59:421-37.

8. Morrison SA, Jesty J. Tissue factor-dependent activation of tritium-labeled factor IX and factor X in human plasma. Blood. 1984;63:1338-47.

9. Friedman PA, Rosenberg RD, Hauschka PV, Fitz-James A. A spectrum of partially carboxylated prothrombins in the plasmas of coumarin-treated patients. Biochim Biophys Acta. 1977;494:271-6.

10. Perzborn E, Roehrig S, Straub A, Kubitza D, Mueck W, Laux V. Rivaroxaban: a new oral factor Xa inhibitor. Arterioscler Thromb Vasc Biol. 2010;30:376-81.

11. Spyropoulos AC. Investigational treatments of venous thromboembolism. Expert Opin Investig Drugs. 2007;16:431-40.

12. Marler JR, Price TR, Clark GL, Muller JE, Robertson T, Mohr JP, et al. Morning increase in onset of ischemic stroke. Stroke. 1989;20:473-6. 
Figure legend

Fig. 1. Hemorrhage location of the patient taking either warfarin or NOAC. The hemorrhage were seen in cerebral cortex, basal ganglia, and cerebellar nucleus. However, the hemorrhage at basal ganglia seen in DOAC patient were smaller than that of warfarin patients.

Fig. 2. Onset timing of the warfarin-ICH and DOAC-ICH. While warfarin-ICH showed bimodal peak in the morning and evening (Black bar), DOAC-ICH did not show any cluster of onset timing peak.

Table. 1

Baseline characteristics of patients with warfarin-ICH and DOAC-ICH

Table. 2

Clinical outcomes with warfarin-ICH and DOAC-ICH 

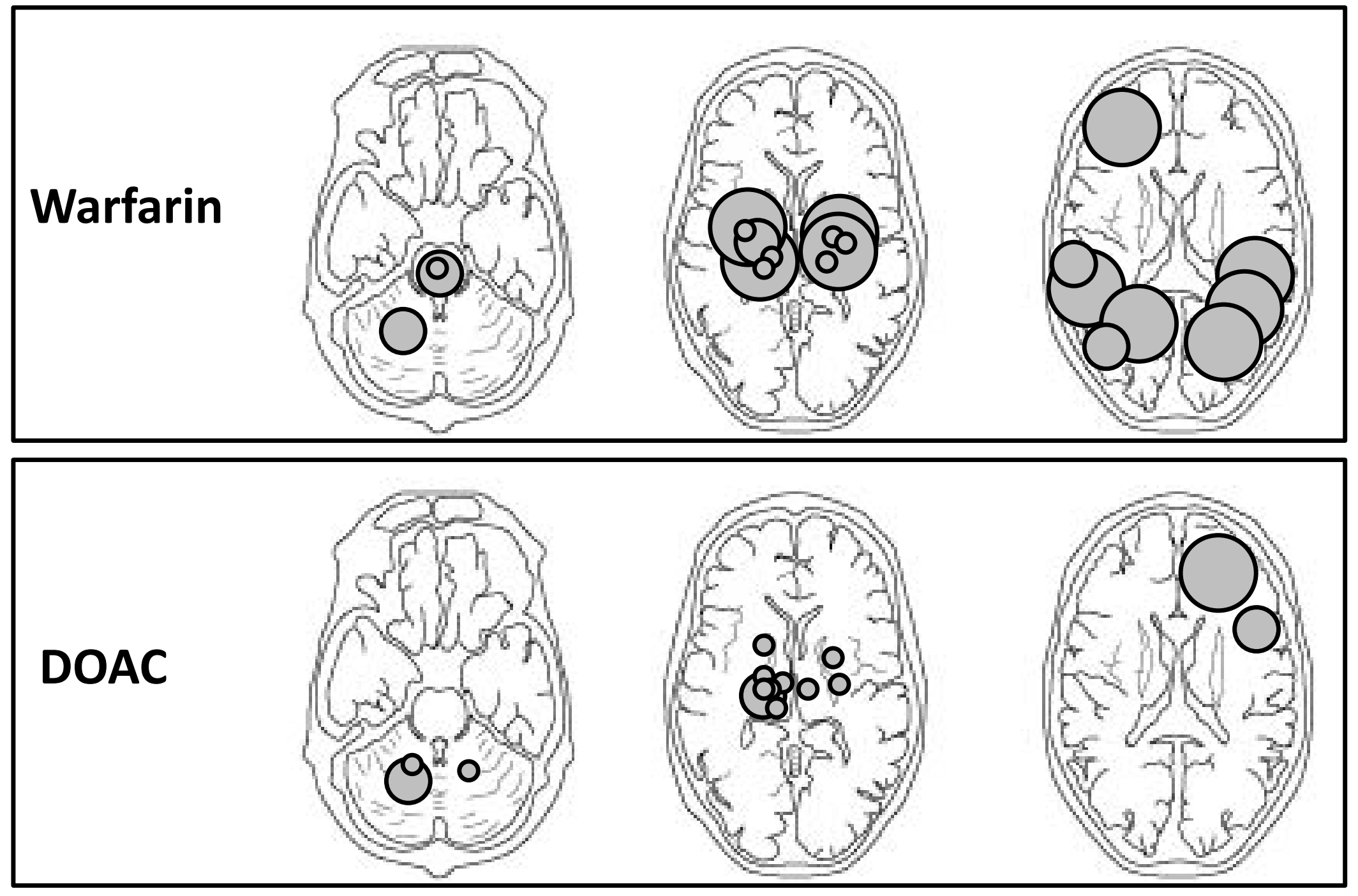

$00-10 \mathrm{ml} \bigcirc 11-30 \mathrm{ml} \bigcirc 31 \mathrm{ml}$


Fig. 2

(n)

4

3

2

1

0

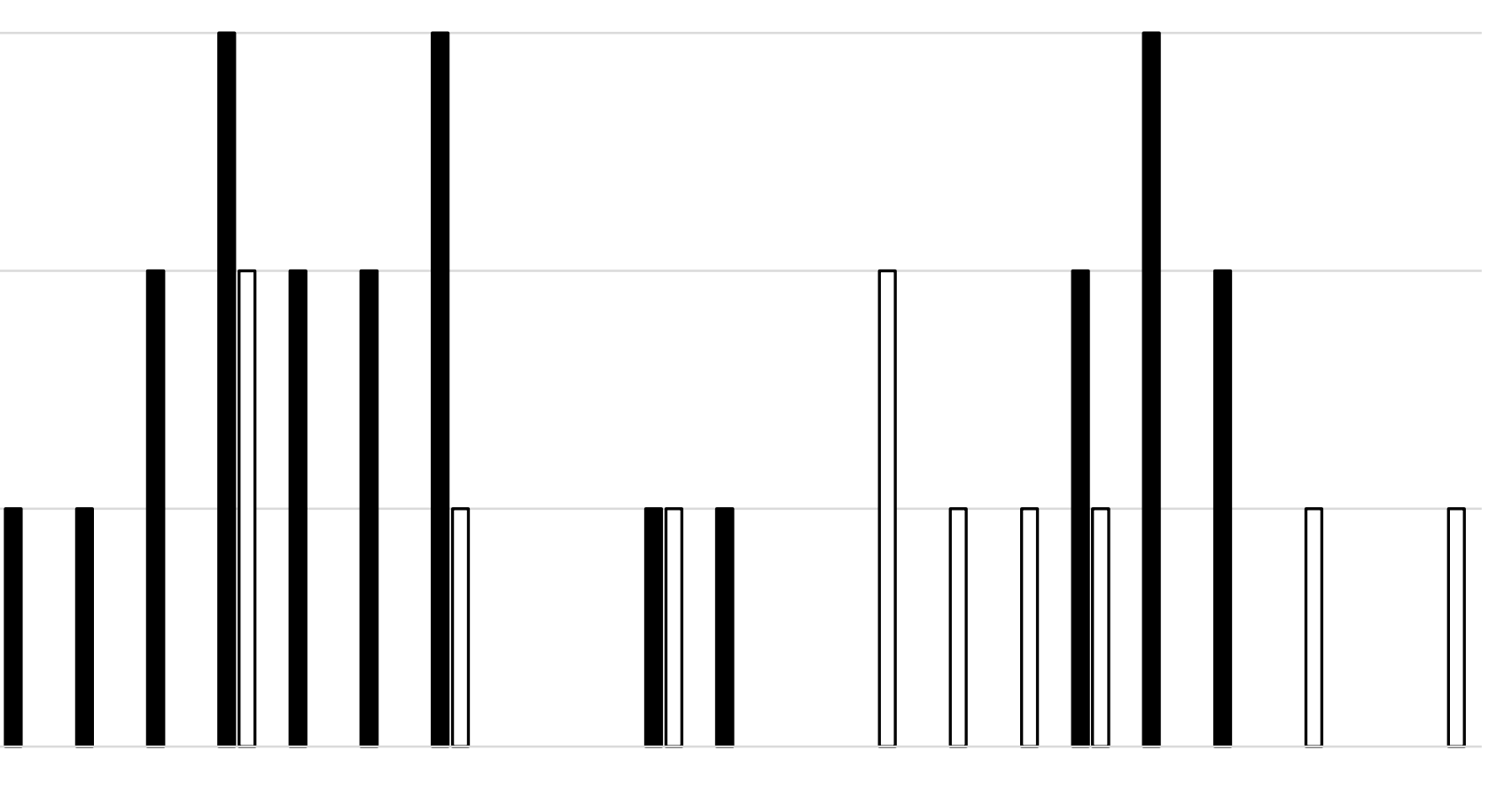

$\begin{array}{llllllllllll}0 & 2 & 4 & 6 & 8 & 10 & 12 & 14 & 16 & 18 & 20 & 22\end{array}$

(hr) 


\begin{tabular}{|c|c|c|c|}
\hline & Warf ari $n-I$ CH $(n=24)$ & DOAC-I CH $(n=15)$ & $p$ val ue \\
\hline Age, y, Medi an (I QR) & $81(75-85)$ & $74(41-81)$ & 0.05 \\
\hline $\mathrm{Mal} e, \mathrm{n}(\%)$ & $14(58 \%)$ & $10(67 \%)$ & 0.34 \\
\hline Anti-coagul ants (dose), $\mathrm{n}(\%)$ & & $\gamma$ varoxaban $(10 \mathrm{mg}), 6(40 \%)$ & \\
\hline & & $\gamma$ varoxaban $(15 \mathrm{mg}), \quad 5(33 \%)$ & \\
\hline & Warfarin $2.9+1.0 \mathrm{mg}$ & Api xaban $(5 \mathrm{mg}), \quad 1(7 \%)$ & \\
\hline & ViarT arth 2. $9 \pm 1.0 \mathrm{mg}$ & Api xaban $(10 \mathrm{mg}), \quad 1(7 \%)$ & \\
\hline & & Edoxaban $(30 \mathrm{mg}), 1(7 \%)$ & \\
\hline & & Edoxaban $(60 \mathrm{mg}), \quad 1(7 \%)$ & \\
\hline PT-I NR medi an (I QR) & 2. $6(2.1-2.9)$ & 1. $2(1.1-1.8)$ & $<0.01$ \\
\hline APTT, medi an (I QR) & $39.0(34.5-47.4)$ & $30.4(26.2-36.6)$ & $<0.01$ \\
\hline Congesti ve Heart Fai I ure, \% & 29. $2 \%$ & $26.6 \%$ & 0.53 \\
\hline Di abet es, $\%$ & 29. $2 \%$ & 20. $0 \%$ & 0.52 \\
\hline Hypertensi on, \% & 87. $5 \%$ & 93. $3 \%$ & 0.55 \\
\hline Chroni c renal fail ure & 17. $4 \%$ & $0.0 \%$ & 0.04 \\
\hline Anem a, $\%$ & $56.5 \%$ & 13. $3 \%$ & $<0.01$ \\
\hline Low total-chol ester ol , $\%$ & 15. $4 \%$ & 26. $3 \%$ & 0.46 \\
\hline Concurrent anti pl atel et, $\%$ & $37.5 \%$ & 13. $3 \%$ & 0.16 \\
\hline & $\begin{array}{c}\text { Aspi ri n ( } 100 \mathrm{mg}), 7(29.2 \%) \\
\text { Ti cl opi di ne (100mg), } 1\end{array}$ & Aspi rin $(100 \mathrm{mg}), \quad 1(6.7 \%)$ & 0.15 \\
\hline & (4. $2 \%)$ & I opi dogrel $(75 \mathrm{mg}), 1(6.7 \%)$ & \\
\hline ant I pl at el ets (dose), n (\%) & $\begin{array}{c}\text { Aspi rin }(100 \mathrm{mg}) \text { and } \\
\text { Cl ost azol }(200 \mathrm{mg}), 1 \\
(4.2 \%)\end{array}$ & & \\
\hline Previ ous i schem c stroke, $\%$ & 25. $0 \%$ & 26. $7 \%$ & 0.66 \\
\hline Previ ous I $\mathrm{CH}$, \% & 8. $3 \%$ & $6.7 \%$ & 0.93 \\
\hline Number of positive T2*, Medi an (I G & $1(1-7)$ & $2(2-11)$ & 0.36 \\
\hline HAS-BLED, medi an (I QR) & $3(2-3)$ & $2(2-3)$ & 0.02 \\
\hline ATR $A$ medi an (I QR) & $4(3-6)$ & $3(1-3)$ & 0.01 \\
\hline ORBI $T$, medi an ( I QR) & $1(1-2)$ & $3(2-4)$ & $<0.01$ \\
\hline
\end{tabular}


Warf ar i $n-I$ OH $(n=24)$ DOAC-I CH $(n=15)$ p val ue

Hemat oma vol ume, Medi an (I QR)

Emergency surgery, \%

Admi ni strati on days, Medi an (I QR)

modi fi ed Ranki n Scal e (mRS), Medi an (

mRS $0-2$ at ti me of di scharge, \%
27. 0 (7. 3-39. 3)

$50 \%$

$31(16-52)$

$5(4-5)$

12. $5 \%$

$5.9(3.5-12.3)<0.01$

$26 \%$

0. 15

$20(14-33)$

0. 20

$2(1-4)$

$<0.01$

$53 \%$

$<0.01$ 